\title{
Geometry of the local equivalence of states
}

\author{
A Sawicki ${ }^{1,2}$ and M Kuś ${ }^{1}$ \\ ${ }^{1}$ Center for Theoretical Physics, Polish Academy of Sciences, Al. Lotników 32/46, 02-668 \\ Warszawa, Poland \\ ${ }^{2}$ School of Mathematics, University of Bristol, University Walk, Bristol BS8 1TW, UK \\ E-mail: assawi@cft.edu.pl, marek.kus@cft.edu.pl
}

\begin{abstract}
.
We present a description of locally equivalent states in terms of symplectic geometry. Using the moment map between local orbits in the space of states and coadjoint orbits of the local unitary group we reduce the problem of local unitary equivalence to an easy part consisting of identifying the proper coadjoint orbit and a harder problem of the geometry of fibers of the moment map. We give a detailed analysis of the properties of orbits of "equally entangled states". In particular we show connections between certain symplectic properties of orbits such as their isotropy and coisotropy with effective criteria of local unitary equivalence.
\end{abstract}

PACS numbers: 03.67.Mn, 03.65.Aa, 02.20.Sv

Submitted to: J. Phys. A: Math. Gen. 


\section{Introduction}

In a recent paper [1] we presented a symplectic description of pure states of composite quantum systems in finite-dimensional Hilbert spaces. In particular we showed that entanglement among subsystems of a multipartite quantum system can be quantified in terms of degeneracy of the canonical symplectic form on the complex projective space restricted to orbits of local, i.e., entanglement preserving unitary groups. In the present paper we would like to continue this line of research by giving a precise geometric description of orbits in low dimensional cases and, above all, by showing how the proposed geometric approach contributes to a solution of an important problem of local unitary equivalence of states.

The classification of states which are connected by local unitary transformations, i.e. operations on the whole system composed from unitary actions (purely quantum evolutions) each of which is restricted to a single subsystem has become recently a topic of several studies [2], [3]. To appreciate the experimental importance of such a setting let us remind that it is a basis for such spectacular applications of quantum information technologies like teleportation or dense codding where the fundamental parts of experiments consist of manipulations restricted to parts of the whole system in distant laboratories.

\section{Symplectic geometry of entanglement}

We start with a short outline of a symplectic description of quantum correlations in composite systems. For details consult [1]. Thorough expositions of the below employed constructions from symplectic geometry can be found in [4] and [5].

\subsection{Space of quantum states as a symplectic manifold}

The Hilbert space of a quantum system consisting of $L$ identical $N$-level systems (qunits) is the tensor product $\$$,

$$
\mathcal{H}=\mathcal{H}_{1} \otimes \cdots \otimes \mathcal{H}_{L}
$$

where each $\mathcal{H}_{k}$ is isomorphic with the complex $N$-dimensional space $\mathbb{C}^{N}$ equipped with the standard Hermitian scalar product $\langle\cdot \mid \cdot\rangle$ (we will denote by the same symbol the standard scalar product in the whole $\mathcal{H}$ as along as it does not lead to confusion).

The set of pure states is the projective space $\mathbb{P}(\mathcal{H})$. We denote a canonical projection from $\mathcal{H}$ to $\mathbb{P}(\mathcal{H})$ by $\pi$ and use the notation $[v]=\pi(v)$ for $v \in \mathcal{H}$.

The projective space $\mathbb{P}(\mathcal{H})$ is equipped with a natural symplectic structure - the Fubini Study form - inherited from the initial Hilbert space $\mathcal{H}$ where a natural symplectic structure is defined in terms of the imaginary part of the scalar product. For further purposes it is convenient to

$\ddagger$ A generalization to nonidentical subsystems, i.e., living in spaces of different dimensionality is straightforward but more tedious. 
calculate the symplectic form on $\mathbb{P}(\mathcal{H})$ in the following way. First observe that the linear action of the unitary group $U(\mathcal{H})$ on $\mathcal{H}$ projects in a natural way to $\mathbb{P}(\mathcal{H})$ as

$$
U[v]:=[U v], \quad U \in U(\mathcal{H}), \quad v \in \mathcal{H}, \quad[v]=\pi(v) .
$$

Let $A \in \mathfrak{u}(\mathcal{H})=\operatorname{Lie}(U(\mathcal{H})$ ) (the Lie algebra of $U(\mathcal{H})$ which also acts linearly on $\mathcal{H}$ ). Denote by $T_{[v]} \mathbb{P}(\mathcal{H})$ the tangent space to $\mathbb{P}(\mathcal{H})$ at the point $[v]$, and by $A_{[v]}$ the vector in $T_{[v]} \mathbb{P}(\mathcal{H})$ tangent to the curve $t \mapsto \pi(\exp (t A) v)$. When $A$ runs through the whole Lie algebra $\mathfrak{u}(\mathcal{H})$ the corresponding $A_{[v]}$ span $T_{[v]} \mathbb{P}(\mathcal{H})$ and the symplectic form on $\mathbb{P}(\mathcal{H})$ at $[v]$ reads

$\omega_{[v]}\left(A_{[v]}, B_{[v]}\right)=\operatorname{Im} \frac{\langle A v \mid B v\rangle\langle v \mid v\rangle-\langle A v \mid v\rangle\langle v \mid B v\rangle}{\langle v \mid v\rangle^{2}}=-\frac{i}{2} \frac{\langle[A, B] v \mid v\rangle}{\langle v \mid v\rangle}, \quad A, B \in \mathfrak{u}(\mathcal{H})$,

where $[\cdot, \cdot]$ is the Lie bracket (commutator) in $\mathfrak{u}(\mathcal{H})$. One checks that indeed $\omega$ is nondegenerate and closed, $d \omega=0$, on $T \mathbb{P}(\mathcal{H})$ and as such makes $\mathbb{P}(\mathcal{H})$ a symplectic manifold. Moreover, as it is clear from the above construction, $\omega$ is invariant with respect to the action (2) of $U(\mathcal{H})$. In other words the action of $U(\mathcal{H})$ on $\mathbb{P}(\mathcal{H})$ is symplectic.

\subsection{Symplectic group actions. Moment map}

Symplectic actions of semisimple groups lead to another important construction useful in our analysis - the moment map. Let a compact semisimple group $G$ acts on a symplectic manifold $(M, \omega)$ via symplectomorhophisms $G \times M \ni(g, x) \mapsto \Phi_{g}(x) \in M$, i.e., we demand that the pullback of the form $\omega$ by $\Phi_{g}$ is the form $\omega$ itself, $\Phi_{g}^{*} \omega=\omega$. For an arbitrary $\xi \in \mathfrak{g}=\operatorname{Lie}(G)$

(the Lie algebra of $G$ ) we define a vector field $\hat{\xi}$ (called in the following the fundamental vector field corresponding to $\xi$ ),

$$
\hat{\xi}(x)=\left.\frac{d}{d t}\right|_{t=0} \Phi_{\exp t \xi}(x) .
$$

Since $G$ acts on $M$ by symplectomorhophisms there exists a function $\mu_{\xi}: M \rightarrow \mathbb{R}$ such that

$$
d \mu_{\xi}=\imath_{\hat{\xi}} \omega:=\omega(\hat{\xi}, \cdot) .
$$

It can be chosen to be linear in $\xi$, i.e., there exists $\mu(x)$ in the space of linear forms on $\mathfrak{g}$ (the dual space to $\mathfrak{g}$ denoted in the following by $\mathfrak{g}^{*}$ ) such that

$$
\mu_{\xi}(x)=\langle\mu(x), \xi\rangle, \quad \mu(x) \in \mathfrak{g}^{*},
$$

where $\langle$,$\rangle is the pairing between \mathfrak{g}$ and $\mathfrak{g}^{*}$. In this way we obtain a function $\mu: M \rightarrow \mathfrak{g}^{*}$ called the moment map.

The group $G$ acts on its Lie algebra $\mathfrak{g}$ via the adjoint action,

$$
\operatorname{Ad}_{g} \xi=\left.\frac{d}{d t}\right|_{t=0} g \exp t \xi g^{-1}=: g \xi g^{-1}, \quad g \in G, \quad \xi \in \mathfrak{g},
$$

which dualizes to the coadjoint action on $\mathfrak{g}^{*}$,

$$
\left\langle\operatorname{Ad}_{g}^{*} \alpha, \xi\right\rangle=\left\langle\alpha, \operatorname{Ad}_{g^{-1}} \xi\right\rangle=\left\langle\alpha, g^{-1} \xi g\right\rangle,
$$


for $g \in G, \xi \in \mathfrak{g}$, and $\alpha \in \mathfrak{g}^{*}$. Under our assumption of the semisimplicity of $G$ the momentum map can be chosen equivariant, i.e., for each $x \in M$ and $g \in G$,

$$
\mu\left(\Phi_{g}(x)\right)=\operatorname{Ad}_{g}^{*} \mu(x)
$$

is fulfilled.

Coadjoint orbits, i.e., the orbits of a coadjoint action of $G$ on $\mathfrak{g}^{*}$ bear a canonical symplectic structure - the so called Kirillov-Kostant-Souriau form. Let $\Omega_{\alpha}$ be the coadjoint orbit going through $\alpha \in \mathfrak{g}^{*}$,

$$
\Omega_{\alpha}=\left\{\operatorname{Ad}_{g}^{*} \alpha: g \in G\right\} .
$$

For any $\xi \in \mathfrak{g}$ let $\tilde{\xi}$ be a vector tangent at $\alpha$ to the curve $t \mapsto \operatorname{Ad}_{\exp (t \xi)}^{*} \alpha$,

$$
\tilde{\xi}=\left.\frac{d}{d t}\right|_{t=0} \operatorname{Ad}_{e x p(t \xi)}^{*} \alpha .
$$

When $\xi$ runs over the whole algebra $\mathfrak{g}$ such vectors span the tangent space to $\Omega_{\alpha}$ at the point $\alpha$. We define the desired symplectic form $\tilde{\omega}$ at the point $\alpha$ by its action on two vectors constructed via (11) from the $\xi$ and $\eta$ elements of $\mathfrak{g}$,

$$
\tilde{\omega}_{\alpha}(\tilde{\xi}, \tilde{\eta})=\langle\alpha,[\xi, \eta]\rangle \text {. }
$$

We can obviously repeat the construction at each point $\beta$ on $\Omega_{\alpha}$ obtaining thus a symplectic form on the whole orbit. It can be checked that $\tilde{\omega}$ constructed in this way is indeed closed and nondegenerate on $\Omega_{\alpha}$, as well as $G$-invarint, i.e., $\left(A d_{g}^{*}\right)^{*} \tilde{\omega}=\tilde{\omega}$.

Due to the equivariance of the moment map (9) the orbit of the $G$-action on $M$ going through a point $x$,

$$
\mathcal{O}_{x}:=\left\{\Phi_{g}(x), g \in G\right\}
$$

is mapped by $\mu$ onto a coadjoint orbit,

$$
\Omega_{\mu(x)}=\left\{\operatorname{Ad}_{g}^{*} \mu(x), g \in G\right\} .
$$

Moreover the map $\mu$ intertwines the symplectic structures on $M$ and coadjoint orbits; if we pull back $\tilde{\omega}$ from $\Omega_{\mu(x)}$ by $\mu$ to $M$ we recover the restriction of $\omega$ to $\mathcal{O}_{x}$,

$$
\mu^{*} \tilde{\omega}=\left.\omega\right|_{\mathcal{O}_{x}}
$$

In this way we obtained a map between two symplectic structures which can be used to investigate properties of $G$-orbits in $M$. First natural questions which can be addressed with the help of the above constructions concerns symplecticity of orbits. In general the moment map does not map $\mathcal{O}_{x}$ onto $\Omega_{\mu(x)}$ diffeomorphically. If it were the case then all $G$-orbits in $M$ would be symplectic, i.e., the restriction of $\omega$ to an orbit would be nondegenerate. To characterize fully the situation when it is the case let us consider two subgroups of $G$ - the stabilizers of, respectively, $x$ and $\mu(x)$,

$$
\begin{aligned}
& \operatorname{Stab}(x)=\left\{g \in G: \Phi_{g}(x)=x\right\} \\
& \operatorname{Stab}(\mu(x))=\left\{g \in G: \operatorname{Ad}_{g}^{*} \mu(x)=\mu(x)\right\} .
\end{aligned}
$$


As a consequence of the equivariance of $\mu$ we have always $\operatorname{Stab}(x) \subset \operatorname{Stab}(\mu(x))$. The KostantSternberg theorem [6] states that an $G$ orbit is symplectic if and only if both stabilizers are equal. As a corollary we obtain that the degeneracy subspace at $x$ defined as

$$
\mathcal{D}_{x}=\left\{u \in T_{x} \mathcal{O}_{x}:\left.\omega\right|_{\mathcal{O}_{x}}(u, v)=0 \quad \forall v \in T_{x} \mathcal{O}_{x}\right\} .
$$

has the dimension

$$
D(x)=\operatorname{dim}\left(\mathcal{D}_{x}\right)=\operatorname{dim}(\operatorname{Stab}(\mu(x)))-\operatorname{dim}(\operatorname{Stab}(x)),
$$

or, taking into account that $\operatorname{dim}(\operatorname{Stab}(x))=\operatorname{dim}(G)-\operatorname{dim}\left(\mathcal{O}_{x}\right)$ and $\operatorname{dim}(\operatorname{Stab}(\mu(x)))=$ $\operatorname{dim}(G)-\operatorname{dim}\left(\Omega_{\mu(x)}\right)$,

$$
D(x)=\operatorname{dim}\left(\mathcal{D}_{x}\right)=\operatorname{dim}\left(\mathcal{O}_{x}\right)-\operatorname{dim}\left(\Omega_{\mu(x)}\right) .
$$

The dimension (20) is of course constant along the whole orbit.

\subsection{Orbits in the space of states. Entanglement}

For the entanglement problem of $L$ identical $N$-level subsystems the relevant group $G$ is the $L$-fold direct product of the special unitary group,

$$
G=S U(N) \times \cdots \times S U(N),
$$

acting in the natural way on the tensor product $\mathcal{H}$, i.e., $g \cdot v=U_{1} v_{1} \otimes \cdots \otimes U_{L} v_{L}$ for $g=\left(U_{1}, \ldots, U_{L}\right) \in G, v=v_{1} \otimes \cdots \otimes v_{L}, v_{k} \in \mathcal{H}_{k}$. This action is projected to the symplectic manifold

$$
M=\mathbb{P}(\mathcal{H}) .
$$

$G$ is a group of local unitary transformations where each $S U(N)$ represents unitary quantum operations exercised on a single subsystem placed in one laboratory. They preserve quantum correlations among subsystems, i.e., they leave the "amount of entanglement" in the system intact.

The moment map for the action of the unitary group $U(\mathcal{H})$ (isomorphic to $U\left(N^{L}\right)$ ) on $\mathbb{P}(\mathcal{H})$ is easily calculated as

$$
\langle\mu([v]), A\rangle=\frac{i}{2} \frac{\langle v \mid A v\rangle}{\langle v \mid v\rangle}, \quad A \in \mathfrak{u}(\mathcal{H}) .
$$

The group $G$ of local transformations (21) is a subgroup of $U(\mathcal{H})$. All relevant formulas for the symplectic forms and the moment map remain the same after appropriate restrictions to $G$ and its Lie algebra $\mathfrak{g}=\mathfrak{s u}(N) \oplus \cdots \oplus \mathfrak{s u}(N)$.

In [1] we showed that the only symplectic orbit in $\mathbb{P}(\mathcal{H})$ is the manifold of separable (nonentangled) states and the dimension $\$$ of degeneracy space $\mathcal{D}_{[v]}$ can be used to quantify entanglement of a state $[v]$ or, in other words, $D([v])$ is an entanglement measure.

$\S$ Here and in the following by dim we understand always the real dimension of the corresponding linear spaces and manifolds, even if they bear complex structures. 


\section{Local unitary equivalence of states}

For simplicity, in the following, as it is customary, we will use the term "states" also for vectors from $\mathcal{H}$ remembering, however, that in fact we have in mind their projections to $\mathbb{P}(\mathcal{H})$.

Two pure states $v, w \in \mathcal{H}$ are called locally unitary (LU) equivalent if and only if there exist $U_{i} \in S U(N)$ such that

$$
[v]=U_{1} \otimes \cdots \otimes U_{L}[w]
$$

i.e., $[u]$ and $[w]$ belong to the same orbit of the action of $G$ on $\mathbb{P}(\mathcal{H})$.

We will show how the above outlined symplectic description of entanglement can help in analyzing local unitary equivalence. As a first step let us calculate the image of an arbitrary state $[v]$ under the moment map (23)

Choosing an orthonormal basis $\left\{e_{k}\right\}, k=1, \ldots, n$ in $\mathcal{H}_{i} \simeq \mathbb{C}^{N}$ we can write an arbitrary $v \in \mathcal{H}$ in the form

$$
v=\sum_{k_{1}, \ldots, k_{L}} C_{k_{1} \ldots k_{L}} e_{k_{1}} \otimes \cdots \otimes e_{k_{L}}
$$

Without losing generality we can assume that $v$ has the unit length.

The Lie algebra $\mathfrak{g}=\mathfrak{s u}(N) \oplus \cdots \oplus \mathfrak{s u}(N)$ is spanned by the matrices $X_{1} \otimes \cdots \otimes I, \ldots$, $I \otimes \cdots \otimes X_{k} \otimes \cdots \otimes I, \ldots, I \otimes \cdots \otimes X_{L}$ with antihermitian $X_{k}$. The dual $\mathfrak{g}^{*}$ can be identified with $\mathfrak{g}$ via the invariant bilinear form $(X, Y)=-\operatorname{tr}(X Y), X, Y \in \mathfrak{g}$, i.e., $\mathfrak{g}^{*} \ni \alpha \sim X \in \mathfrak{g}$ if $\langle\alpha, Y\rangle=-\operatorname{tr}(X Y)$ for an arbitrary $Y \in \mathfrak{g}$. For convenience we supplement this identification by multiplication of $X$ by the imaginary unit making elements of $\mathfrak{g}^{*}$ Hermitian. This is irrelevant for the whole reasoning but allows to treat elements of $\mathfrak{g}^{*}$ as physical observables. Let thus

$$
X=X_{1} \otimes \cdots \otimes I+\ldots+I \otimes \cdots \otimes X_{k} \otimes \cdots \otimes I+\ldots+I \otimes \cdots \otimes X_{L}
$$

be an element of $\mathfrak{g}^{*}$. A straightforward calculation gives

$$
\mu_{X}([v])=\sum_{k}^{L} \sum_{n, m}^{N} C_{m n}^{(k)}\left\langle e_{m} \mid X_{k} e_{n}\right\rangle,
$$

where

$$
C_{n m}^{(k)}=\sum_{l_{1}, \ldots, l_{L-1}} \bar{C}_{l_{1} \ldots n \ldots l_{L-1}} C_{l_{1} \ldots m \ldots l_{L-1}},
$$

where the overbar denotes the complex conjugation and the summation is over all corresponding pairs of indices except those on the $k$-th places. The positive semidefinite matrices $C^{(k)}$ are in fact the reduced density matrices of the subsystems. In the following we will occasionally use the notation $C^{(k)}([v])$ to exhibit explicitly the dependence of the reduced density matrices on the original state $[v]$.

It is known (see e.g., [5]) that each coadjoint orbit of a compact group (such as our $G$ ) intersects the dual $\mathfrak{t}^{*}$ to the maximal commutative subalgebra $\mathfrak{t}$ of $\mathfrak{g}$. In our case $\mathfrak{t}^{*}$ is spanned by 
$I \otimes \cdots \otimes Y_{k} \otimes \cdots \otimes I$ with diagonal Hermitian $Y_{k}$. In general a coadjoint orbit intersects $\mathfrak{t}^{*}$ in several points connected by elements of the Weyl group. By restricting to a particular Weyl chamber, e.g., by demanding that the diagonal elements of the diagonal matrices $Y_{k}$ appear in the nonincreasing order, we get rid of this redundancy.

In [1] it was shown that for a state $[u]$ its image under the moment map $\mu([u])$ belongs to $t^{*}$ if and only if all matrices $C^{(k)}([u])$ are diagonal, $C^{(k)}([u])=\operatorname{diag}\left(p_{1 k}^{2}, \ldots, p_{N k}^{2}\right)$, and we have in this case,

$$
\mu([u])=Y_{1} \otimes I \otimes \cdots \otimes I+\cdots+I \otimes \cdots \otimes I \otimes Y_{L},
$$

where, up to an irrelevant multiplicative constant,

$$
Y_{k}=\operatorname{diag}\left(-\frac{1}{N}+p_{1 k}^{2}, \ldots,-\frac{1}{N}+p_{N k}^{2}\right) .
$$

Since the orbit $\mathcal{O}_{[v]}$ is mapped by the moment map onto the coadjoint orbit $\Omega_{\mu([v])}$ each $v \in \mathcal{H}$ can be transformed by some group element $U_{1} \otimes \ldots \otimes U_{L} \in G$ to $v^{\prime} \in \mathcal{H}$ such that the moment map $\mu\left(\left[v^{\prime}\right]\right)$ belongs to $\mathfrak{t}^{*}$, i.e, the reduced density matrices $C^{(k)}\left(\left[v^{\prime}\right]\right)$ are diagonal, $C^{(k)}\left(\left[v^{\prime}\right]\right)=\operatorname{diag}\left(p_{1 k}^{2}, \ldots, p_{N k}^{2}\right), p_{1 k}^{2} \geq \ldots \geq p_{N k}^{2}$. The corresponding $U_{k}$ are recovered from the matrices diagonalizing the reduced density matrices of the state $v$. If $\tilde{U}_{k}^{\dagger} C^{(k)}([v]) \tilde{U}_{k}=C^{(k)}\left(\left[v^{\prime}\right]\right)$ then $U_{k}=\tilde{U}_{k}^{T}\left({ }^{T}\right.$ denotes the transposition). Such a state $x^{\prime}=\left[v^{\prime}\right]$ is called in [2, 3] the 'sorted trace decomposition' of the state $x=[v]$. Observe that in the case of two subsystems $(L=2)$, the transformation form $x$ to $x^{\prime}$ can be made unique using the Schmidt decomposition of a bipartite state.

Let us now return to the question of the local unitary equivalence of two states $x=[u]$ and $y=[w], x, y \in \mathbb{P}(\mathcal{H})$. Obviously, the equalities $C^{(k)}(x)=C^{(k)}(y), k=1, \ldots, L$, give a necessary condition for the local unitary equivalence of $x$ and $y$. If they are fulfilled we can use matrices $\tilde{U}_{k}$ and $\tilde{V}_{k}$ diagonalizing, respectively, $C^{(k)}(x)$ and $C^{(k)}(y)$ to transform $x$ and $y$ to their sorted trace decompositions $x^{\prime}$ and $y^{\prime}$. The equality $x^{\prime}=y^{\prime}$ is then a sufficient condition for the local equivalence of $x=[v]$ and $y=[w]$ and, explicitly,

$$
[v]=U_{1}^{\dagger} V_{1} \otimes \cdots \otimes U_{L}^{\dagger} V_{L}[w] .
$$

It is also clear that if the spectra of reduced density matrices for $x$ and $y$ are equal but $x^{\prime} \neq y^{\prime}$ the states $x$ and $y$ may still be locally unitary equivalent. Indeed, the equality of spectra of the reduced density matrices of $x$ and $y$ means that $\mu\left(x^{\prime}\right)=\mu\left(y^{\prime}\right)$. If $y^{\prime}=\Phi_{g^{\prime}}\left(x^{\prime}\right)$ for some $g^{\prime} \in G$, which is equivalent to $y=\Phi_{g}(x)$ for some $g \in G$, then due to the equivariance of $\mu$,

$$
\mu\left(x^{\prime}\right)=\mu\left(y^{\prime}\right)=\mu\left(\Phi_{g^{\prime}}\left(x^{\prime}\right)\right)=\operatorname{Ad}_{g^{\prime}}^{*}\left(\mu\left(x^{\prime}\right)\right),
$$

i.e., $g^{\prime} \in \operatorname{Stab}\left(\mu\left(x^{\prime}\right)\right)$. Summarizing, $x=[v]$ and $y=[w]$ with the same spectra of reduced density matrices are equivalent if and only if there exists $g^{\prime} \in \operatorname{Stab}\left(\mu\left(x^{\prime}\right)\right)$ such that $\Phi_{g^{\prime}}\left(x^{\prime}\right)=y^{\prime}$. Since $\operatorname{Stab}\left(\mu\left(x^{\prime}\right)\right) \supset \operatorname{Stab}\left(x^{\prime}\right)$ this can happen also for $x^{\prime} \neq y^{\prime}$.

In a generic case when spectra of all reduced density matrices are nondegenerate (there are no multiple eigenvalues) the stabilizer $\operatorname{Stab}\left(\mu\left(x^{\prime}\right)\right)$ consists of diagonal unitary matrices, as it is clear from Eq.(30) giving explicitly the value of the moment map at an intersection with $\mathfrak{t}^{*}$. 
In this case the algorithm of deciding the local unitary equivalence can be effectively applied. In nongeneric cases $\operatorname{Stab}\left(\mu\left(x^{\prime}\right)\right)$ is a subgroup of $G=S U(N)^{\otimes L}$. As long as it is a proper subgroup the effort of checking local unitary equivalence can be considerably eased, but if for

all $k$ we have $C^{(k)}\left(x^{\prime}\right)=\frac{1}{\sqrt{N}} I$ ('maximally entangled states') we return to the full group $G$ since in this case $\operatorname{Stab}\left(\mu\left(x^{\prime}\right)\right)=S U(N) \otimes \cdots \otimes S U(N)$.

\section{Fibers of the moment map}

From the preceding section it is clear that to make progress in checking the local unitary equivalence we have to investigate closer the fiber of the moment map at $x$ (we will omit ' in the following assuming that $x$ is already reduced to its sorted trace form), i.e.

$$
\mathcal{F}_{x}:=\{z \in M: \mu(z)=\mu(x)\}=\mu^{-1}(\mu(x)) .
$$

Let $\left\{\xi_{k}\right\}, k=1, \ldots, d=\operatorname{dim} \mathfrak{g}$, be a basis in the Lie algebra $\mathfrak{g}$. The corresponding vector fields $\hat{\xi}_{k}$ at $x$ (c.f. Eq. (4) ) span the tangent space $T_{x} \mathcal{O}_{x}$ to the orbit through $x$ at $x$. On the other hand, the fiber $\mathcal{F}_{x}$ is a common level set of the functions $\mu_{\xi_{k}}$,

$$
\mathcal{F}_{x}=\left\{z \in M: \mu_{\xi_{k}}(z)=c_{k}\right\}, \quad c_{k}=\mu_{\xi_{k}}(x), \quad k=1, \ldots, d
$$

Let us define:

$$
\operatorname{Ker}_{x}(d \mu):=\left\{a \in T_{x} M: d \mu_{\xi_{k}}(x)(a)=0, k=1, \ldots, d\right\}
$$

From (5) we have

$$
d \mu_{\xi_{k}}(a)=\omega\left(\hat{\xi}_{k}, a\right)
$$

hence $a \in \operatorname{Ker}_{x}(d \mu)$ if and only if $a$ is $\omega$-orthogonal to all $\hat{\xi}_{k}$ and since the latter span $T_{x} \mathcal{O}_{x}$ we obtain

$$
\operatorname{Ker}_{x}(d \mu)=\left(T_{x} \mathcal{O}_{x}\right)^{\perp \omega}
$$

Since (c.f. 34), $\mu_{\xi_{k}}$ are constant on $\mathcal{F}$, we have $d \mu_{\xi_{k}}(a)=0$ for $a \in T_{x} \mathcal{F}_{x}$. Hence $T_{x} \mathcal{F}_{x} \subset \operatorname{Ker}_{x}(d \mu)$ and finally $T_{x} \mathcal{F}_{x} \subset\left(T_{x} \mathcal{O}_{x}\right)^{\perp \omega}$.

It is obvious that the above reasoning does not depend on the choice of a particular point in $\mathcal{F}_{x}$, i.e.,

$$
T_{y} \mathcal{F}_{x} \subset\left(T_{y} \mathcal{O}_{x}\right)^{\perp \omega}, \quad y \in \mathcal{F}_{x}
$$

A submanifold $P$ of a symplectic manifold $M$ is called coisotropic if for arbitrary $y \in P$ we have $\left(T_{y} P\right)^{\perp \omega} \subset T_{y} P$. We conclude thus that if $\mathcal{O}_{x}$ is coisotropic then $\mathcal{F}_{x} \subset \mathcal{O}_{x}$. Indeed from (38) and the cosisotropy of $\mathcal{O}_{x}$ at each $y \in \mathcal{F}_{x}$ we have $T_{y} \mathcal{F}_{x} \subset T_{y} \mathcal{O}_{x}$. Hence, in this case examining whether some $y$ belongs to $\mathcal{O}_{x}$ (and, consequently whether $y$ and $x$ are LU-equivalent) reduces to checking if their sorted trace forms $x^{\prime}$ and $y^{\prime}$ have the same image under the moment map. The coisotropy of $\mathcal{O}_{x}$ is a sufficient but not necessary condition for $\mathcal{F}_{x} \subset \mathcal{O}_{x}$, since even for a non-coisotropic orbit the fiber can be fully contained in it. 
Summarizing the reasonings presented in the preceding sections we may formulate the following observations. Let us assume that for two states $x=[v]$ and $y=[w]$ the necessary condition for LU equivalence is fulfilled, i.e., the spectra of the reduced density matrices $C^{k}(x)$ and $C^{k}(y)$ are equal for all $k=1, \ldots, L$ and let $x^{\prime}=\left[v^{\prime}\right]$ and $y^{\prime}=\left[w^{\prime}\right]$ be the sorted trace forms of $x$ and $y$. Then,

(i) If the spectra of $C^{k}(x)$ for all $k$ are non-degenerate then establishing the LU equivalence of $x$ and $y$ consist of checking whether tere exists a diagonal unitary $U$ such that $\left[v^{\prime}\right]=\left[U w^{\prime}\right]$ which reduces to a straightforward calculation.

(ii) If some spectra of the reduced density matrices are degenerate the states are LU equivalent if the fiber of the moment map $\mathcal{F}_{x}$ is contained in the orbit $\mathcal{O}_{x}$. A sufficient but not necessary condition for such an inclusion is the coisotropy of the orbit $\mathcal{O}_{x}$.

In the two-partite case $(L=2)$ the LU equivalence is easily checked by performing the Schmidt decomposition of both considered states. If the non-zero Schmidt coefficients, equal to the square roots of the reduced density matrices (in this case equal for both subsystems) are equal, the states are LU equivalent. This simple criterion is reflected in the geometry of orbits and fibers of the moment map, albeit not in the simplest possible way consisting of the coisotropy of orbits. In the next section we give a detailed analysis of $L=2$ case identifying coisotropic and non-coisotropic orbits and showing that also the latter contain the whole corresponding fibers of the moment map.

\section{Two-partite states}

As already mentioned, for $L=2$ the reduction of a state $x=[v]$ to its sorted trace form gives the Schmidt decomposition of $v$. We will assume that this operation has been already performed, hence we assume that $v$ reads as

$$
v=\sum_{k=1}^{N} p_{k} e_{k} \otimes f_{k}
$$

where $\left\{e_{k}\right\}$ and $\left\{f_{k}\right\}$ are appropriate orthonormal bases in $\mathbb{C}^{N}$. Let us denote by $m_{0}$ the number of vanishing $p_{k}$ and by $m_{l}$ the multiplicity of the consecutive nonzero coefficients $p_{k}$, thus $\sum_{l=0}^{r} m_{l}=N$, where $r$ is the number of different nonvanishing coefficients in the Schmidt decomposition (39).

It was proved in [1] (see also [7]) that the dimensions of orbits $\mathcal{O}_{[v]}$ and $\Omega_{\mu([v])}$ are given as,

$$
\begin{aligned}
\operatorname{dim}\left(\mathcal{O}_{[v]}\right) & =2 N^{2}-2 m_{0}^{2}-\sum_{n=1}^{r} m_{n}^{2}-1, \\
\operatorname{dim}\left(\Omega_{\mu([v])}\right) & =2 N^{2}-2 \sum_{l=0}^{r} m_{n}^{2} .
\end{aligned}
$$

From the above we can thus easily calculate the dimension of the space $\omega$-orthogonal to $T_{[v]} \mathcal{O}_{[v]}$

$$
\operatorname{dim}\left(\left(T_{[v]} \mathcal{O}_{[v]}\right)^{\perp \omega}\right)=\operatorname{dim}(\mathbb{P}(\mathcal{H}))-\operatorname{dim}\left(\mathcal{O}_{[v]}\right)=\left(2 N^{2}-2\right)-\operatorname{dim}\left(\mathcal{O}_{[v]}\right)=2 m_{0}^{2}-\sum_{n=1}^{r} m_{n}^{2}-1
$$


and the dimension of the degeneracy space (see Eq. (201)),

$$
D([v])=\operatorname{dim}\left(\mathcal{O}_{[v]}\right)-\operatorname{dim}\left(\Omega_{\mu([v])}\right)=\sum_{n=1}^{r} m_{n}^{2}-1 .
$$

Observe that the degeneracy space (18) consists of exactly those vectors from $T_{[v]} \mathcal{O}_{[v]}$ which simultaneously belong to $\left(T_{[v]} \mathcal{O}_{[v]}\right)^{\perp \omega}$ hence it is the part of $\left(T_{[v]} \mathcal{O}_{[v]}\right)^{\perp \omega}$ contained in $T_{[v]} \mathcal{O}_{[v]}$. Comparing (42) and (43) we infer that an orbit is coisotropic if and only if all coefficients in the Schmidt decomposition (39) differ from zero. In this case, as we showed above, fibers of the moment map are contained in the corresponding orbits. We will prove that this is the case also for non-coisotropic orbits.

First, observe that we have the following direct sum decomposition of subspaces

$$
\left(T_{[v]} \mathcal{O}_{[v]}\right)^{\perp \omega}=\mathcal{D}_{[v]} \oplus S
$$

where $\mathcal{D}_{[v]}$ is the degeneracy space (18) and $S$ is a symplectic subspace of dimension $2 m_{0}^{2}$ spanned by $e_{k} \otimes f_{l}$ and $i e_{k} \otimes f_{l}$, where $k$ and $l$ are such that the corresponding $p_{k}$ and $p_{l}$ in (39) vanish $\amalg$. The symplecticity of $S$ is obvious since $\omega$ is nondegenerate on it. Checking that $S$ is indeed spanned by the mentioned vectors is a matter of a short calculation. Let us notice first that for any $e_{k} \otimes f_{l}$ and $i e_{k} \otimes f_{l}$ such that $p_{k}=0=p_{l}$ in (39) we have

$$
\left\langle e_{k} \otimes f_{l} \mid v\right\rangle=0=\left\langle i e_{k} \otimes f_{l} \mid v\right\rangle, \quad\left\langle e_{k} \otimes f_{l} \mid e_{k} \otimes f_{l}\right\rangle=1=\left\langle i e_{k} \otimes f_{l} \mid i e_{k} \otimes f_{l}\right\rangle,
$$

which means that $e_{k} \otimes f_{l}$ and $i e_{k} \otimes f_{l}$ belong to $\in T_{[v]} M$. On the other hand (see the remark

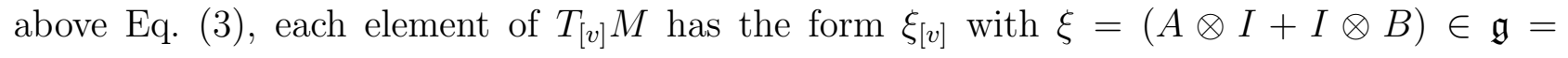
$\mathfrak{s u}(N) \otimes \mathfrak{s u}(N)$. Using formula (3) and (45) we see that

$$
\omega_{[v]}\left(e_{k} \otimes f_{l},(A \otimes I+I \otimes B)_{[v]}\right)=\operatorname{Im}\left\langle e_{k} \otimes f_{l} \mid(A \otimes I+I \otimes B) v\right\rangle,
$$

for any $A, B \in \mathfrak{s u}(N)$. Direct calculations give

$$
\left\langle e_{k} \otimes f_{l} \mid(A \otimes I+I \otimes B) v\right\rangle=\sum_{i=1}^{N} p_{i}\left(\left\langle e_{k} \mid A e_{i}\right\rangle\left\langle f_{l} \mid f_{i}\right\rangle+\left\langle e_{k} \mid e_{i}\right\rangle\left\langle f_{l} \mid B f_{i}\right\rangle\right)
$$

Notice that $\left\langle e_{k} \mid A e_{i}\right\rangle\left\langle f_{l} \mid f_{i}\right\rangle+\left\langle e_{k} \mid e_{i}\right\rangle\left\langle f_{l} \mid B f_{i}\right\rangle \neq 0$ if and only if $i=l$ or $i=k$ but then from our assumption $p_{i}=0$ which means (47) and (46) vanish. Hence, $e_{k} \otimes f_{l}$ and $i e_{k} \otimes f_{l}$ are elements of $S \subset\left(T_{[v]} \mathcal{O}_{[v]}\right)^{\perp \omega}$. Comparing the dimensions of $\operatorname{dim} S=2 m_{0}^{2}$ with (42) and (43) we obtain (44).

It is now enough to show that fibers of the moment map are not tangent to $S$. Let us use again the notation $x=[v]=\pi(v)$ and assume the contrary, i.e., that there exists a curve $t \mapsto x(t) \in \mathcal{F}$ with $x(0)=x$, such that the tangent $\dot{x}(0)$ to it at $x$ belongs to $S$. Since in the two-partite case the fiber is given as a level set of functions $\mu_{I \otimes A_{k}}$, and $\mu_{A_{k} \otimes I}$, where $A_{k} \operatorname{span} \mathfrak{s u}(N)$ it follows that for an arbitrary $A \in \mathfrak{s u}(N)$ we have $\mu_{I \otimes A}(x(t))=$ const, $\mu_{A \otimes I}(x(t))=$ const and, $\|$ Remember that we treat $T_{[v]} M$ as a real vector space, hence $e_{k} \otimes f_{l}$ and $i e_{k} \otimes f_{l}$ are different vectors. 
consequently,

$$
\begin{aligned}
& \left.\frac{d \mu_{I \otimes A}(x(t))}{d t}\right|_{t=0}=0=\left.\frac{d \mu_{A \otimes I}(x(t))}{d t}\right|_{t=0}, \\
& \left.\frac{d^{2} \mu_{I \otimes A}(x(t))}{d t^{2}}\right|_{t=0}=0=\left.\frac{d^{2} \mu_{A \otimes I}(x(t))}{d t^{2}}\right|_{t=0} .
\end{aligned}
$$

The first condition is always fulfilled due to the definition (5) of $\mu$,

$$
d \mu_{I \otimes A}(\dot{x}(0))=\omega(\widehat{I \otimes A}, \dot{x}(0))=0,
$$

since $\widehat{I \otimes A}$ belongs to $T_{x} \mathcal{O}_{x}$ and from the assumption, $\dot{x}(0) \in S \subset\left(T_{x} \mathcal{O}_{x}\right)^{\perp \omega}$. The condition (49) reads explicitly

$$
\dot{x}(0)^{T} \cdot\left[D^{2} \mu_{I \otimes A}\right] \cdot \dot{x}(0)+D^{1} \mu_{I \otimes A} \cdot \ddot{x}(0)=0,
$$

where $D^{1} \mu_{I \otimes A}$ is the first derivative vector and $\left[D^{2} \mu_{I \otimes A}\right]$ the second derivative matrix of the function $\mu_{I \otimes A}$ at $x$. Since $\dot{x}(0)$ is tangent to $M$ at $[v]$ we have $\dot{x}(0)=B_{[v]}=[B v]$ for some $B \in \mathfrak{s u}\left(N^{2}\right)$ (see again the remark above Eq.(3) $)$ ), by a direct calculation we find

$$
\dot{x}(0)^{T} \cdot\left[D^{2} \mu_{I \otimes A}\right] \cdot \dot{x}(0)=-\frac{i\langle[[I \otimes A, B], B] v \mid v\rangle}{2\langle v \mid v\rangle} .
$$

Our aim now is to show that there exist such an $A \in \mathfrak{s u}(N)$ that (51) is not fulfilled for any choice of $\dot{x}(0) \in S$. In Appendix A we show that the goal is achieved by taking $A \in \mathfrak{s u}(N)$ as a diagonal traceless matrix with $A_{k k}=i$ if $p_{k} \neq 0$ and $A_{k k}=-i \frac{N-m_{0}}{m_{0}}$ if $p_{k}=0$ in (39). Appendix $\mathrm{B}$ contains a complete description of orbits in the simplest non-trivial example of two qutrits $(N=2, L=3)$.

\section{Geometric structure of orbits through GHZ states}

As remarked at the end of Section 3, the method of checking the local unitary equivalence based on comparison of the moment map images gives no advantages when all reduced density matrices are proportional to the identity. In this section we will show how it is reflected in the structure of orbits through the so called Greenberger-Horn-Zeilinger (GHZ) states for $L \geq 3$ qubits. The Hilbert space will be thus $\mathcal{H}=\left(\mathbb{C}^{2}\right)^{\otimes L}$ with the real dimension $\operatorname{dim}(\mathcal{H})=2^{L+1}$ so $\operatorname{dim}(\mathbb{P}(\mathcal{H}))=2^{L+1}-2$. We are interested in orbits of the action of $G=S U(2)^{\times L}$ on $\mathbb{P}(\mathcal{H})$. The Lie algebra $\mathfrak{g}$ of $G$ is spanned by

$$
\begin{gathered}
\mathcal{X}_{k}=i I \otimes \cdots \otimes \underset{\hat{k}}{\sigma_{x}} \otimes \cdots \otimes I \\
\mathcal{Y}_{k}=i I \otimes \cdots \otimes \underset{\sigma_{y}}{\sigma_{k}} \otimes \cdots \otimes I \\
\mathcal{Z}_{k}=i I \otimes \cdots \otimes \underset{\hat{\kappa}}{\sigma_{z}} \otimes \cdots \otimes I \\
{ }_{k}
\end{gathered}
$$

where $\sigma_{x}, \sigma_{y}$, and $\sigma_{z}$ are the Pauli matrices and $k=1, \ldots, L$. The fibers of the moment map are given as level sets of the functions $\mu_{\mathcal{X}_{k}}, \mu_{\mathcal{Y}_{k}}, \mu_{\mathcal{Z}_{k}}, k=1, \ldots, L$. 
Let us consider the $L$-partite GHZ state

$$
v_{L}=\frac{1}{\sqrt{2}}\left(|0\rangle^{\otimes L}+|1\rangle^{\otimes L}\right)
$$

where in order to make the formulas more readable we switched to the customary notation of the qubit states $e_{1}=|0\rangle, e_{2}=|1\rangle$ together with $|k\rangle \otimes|l\rangle=|k l\rangle$ and $|k k \ldots k\rangle=|k\rangle^{\otimes L}$, etc. The matrices $C^{k}\left(\left[v_{L}\right]\right)(28)$ are the same for all $k$,

$$
C^{k}\left(\left[v_{L}\right]\right)=\left(\begin{array}{cc}
\frac{1}{2} & 0 \\
0 & \frac{1}{2}
\end{array}\right) .
$$

For the GHZ states we have $\mu\left(\left[v_{L}\right]\right)=0$. Indeed,

$\left\langle\mu\left(\left[v_{L}\right]\right), I \otimes I \otimes \ldots \otimes X_{k} \otimes \ldots \otimes I\right\rangle=\frac{i}{2}\left\langle v_{L} \mid I \otimes I \otimes \ldots \otimes X_{k} \otimes \ldots \otimes I v_{L}\right\rangle=\frac{i}{4} \operatorname{tr} X_{k}=0$,

for an arbitrary $X_{k} \in \mathfrak{s u}(2)$. Hence, for any two vectors $A_{\left[v_{L}\right]}, B_{\left[v_{L}\right]} \in T_{\left[v_{L}\right]} \mathcal{O}_{\left[v_{L}\right]}$, where $A, B \in \mathfrak{g}$ we have

$$
\omega\left(A_{\left[v_{L}\right]}, B_{\left[v_{L}\right]}\right)=-\frac{i}{2}\left\langle[A, B] v_{L} \mid v_{L}\right\rangle=\left\langle\mu\left(\left[v_{L}\right]\right),[A, B]\right\rangle=0,
$$

since $\mu\left(\left[v_{L}\right]\right)=0$.

Notice that for any $L \geq 3$ (59) implies that $T_{\left[v_{L}\right]} \mathcal{O}_{\left[v_{L}\right]} \subseteq\left(T_{\left[v_{L}\right]} \mathcal{O}_{\left[v_{L}\right]}\right)^{\perp \omega}$. We will show now that the orbit $\mathcal{O}_{\left[v_{3}\right]}$ through $\left[v_{3}\right]$ is Lagrangian $\mathbb{\|}$, i.e., $T_{\left[v_{L}\right]} \mathcal{O}_{\left[v_{L}\right]}=\left(T_{\left[v_{L}\right]} \mathcal{O}_{\left[v_{L}\right]}\right)^{\perp \omega}$ whereas for $L \geq 4$ it is isotropic, i.e., $T_{\left[v_{L}\right]} \mathcal{O}_{\left[v_{L}\right]} \subset\left(T_{\left[v_{L}\right]} \mathcal{O}_{\left[v_{L}\right]}\right)^{\perp \omega}$. In other words for $L=3$ the orbit $\mathcal{O}_{\left[v_{L}\right]}$ is (minimally) coisotropic and for $L>3$ the orbit $\mathcal{O}_{\left[v_{L}\right]}$ is not coisotropic.

The space $T_{\left[v_{L}\right]} \mathcal{O}_{\left[v_{L}\right]}$ is spanned by the vectors

$$
\begin{aligned}
& \mathcal{X}_{k} v_{L}=\frac{i}{\sqrt{2}}(|\underbrace{\ldots}_{k} \ldots 0\rangle+|1 \ldots \underset{k}{0} \ldots 1\rangle), \\
& \mathcal{Y}_{k} v_{L}=\frac{1}{\sqrt{2}}\left(\left|0 \ldots \hat{k}_{k}^{1} \ldots 0\right\rangle-\left|1 \ldots \hat{k}_{k}^{0} \ldots 1\right\rangle\right) \text {, } \\
& \mathcal{Z}_{k} v_{L}=\frac{i}{\sqrt{2}}\left(|0\rangle^{\otimes L}-|1\rangle^{\otimes L}\right),
\end{aligned}
$$

with $k=1, \ldots, L$. The above $2 L+1$ vectors are mutually orthogonal, hence

Fact 1 The orbit $\mathcal{O}_{\left[v_{L}\right]}$ has dimension

$$
\operatorname{dim} \mathcal{O}_{\left[v_{L}\right]}=2 L+1
$$

Since $\frac{1}{2} \operatorname{dim}(\mathbb{P}(\mathcal{H}))=2^{L}-1$, we have as a immediate consequence,

I The Lagrangian subspace $U$ of symplectic space $(V, \omega)$ is the minimally coisotropic $\left(U \subseteq U^{\perp \omega}\right)$ and at the same time maximally isotropic $\left(U \supseteq U^{\perp \omega}\right)$ subspace of $V$, i.e., for any coisotropic space $W \subset U$ we have $W=U$ and for any isotropic space $W^{\prime} \supset U$ we have $W^{\prime}=V$. These conditions imply that $U$ is Lagrangian if and only if $U=U^{\perp \omega}$, hence $\left.\omega\right|_{U}=0$ and $\operatorname{dim} U=\frac{1}{2} \operatorname{dim} V$. 
Fact 2 The orbit $\mathcal{O}_{\left[v_{3}\right]}$ is Lagrangian, and hence the fiber of the moment map is contained inside it. If $L \geq 4$ then $\mathcal{O}_{\left[v_{L}\right]}$ is not Lagrangian.

Indeed from (63) $\operatorname{dim} \mathcal{O}_{\left[v_{3}\right]}=7=2^{3}-1=\frac{1}{2} \operatorname{dim}(\mathbb{P}(\mathcal{H}))$, whereas for $L \geq 4$ we have $2^{L}-1>2 L+1$ hence the orbits have too small dimension to be Lagrangian.

The fact that $\mathcal{O}_{\left[v_{3}\right]}$ is Lagrangian (so also coisotropic), implies that necessary and sufficient condition for two states $[u]$ and $[w]$ of three qubits to belong to $\mathcal{O}_{\left[v_{3}\right]}$ is $\mu([u])=0=\mu([w])$.

For $L \geq 4$ the fiber of the moment map is not entirely contained in $\mathcal{O}_{\left[v_{L}\right]}$. We will show that in fact $T_{\left[v_{L}\right]} \mathcal{F}_{\left[v_{L}\right]}=\left(T_{\left[v_{L}\right]} \mathcal{O}_{\left[v_{L}\right]}\right)^{\perp \omega}$.

Let $A_{\left[v_{L}\right]} \in T_{\left[v_{L}\right]} \mathcal{O}_{\left[v_{L}\right]}$, i.e, $A_{\left[v_{L}\right]}=\left[A v_{L}\right]$ where $A$ is of the form (60)-(62) ,

$$
A=i I \otimes \cdots \otimes \underset{\hat{k}}{\sigma_{\beta}} \otimes \cdots \otimes I, \quad \beta \in\{x, y, z\} .
$$

The space $\left(T_{\left[v_{L}\right]} \mathcal{O}_{\left[v_{L}\right]}\right)^{\perp \omega}$ is spanned by these $B_{\left[v_{L}\right]} \in T_{\left[v_{L}\right]} \mathbb{P}(\mathcal{H})$ for which $\omega_{\left[v_{L}\right]}\left(A_{\left[v_{L}\right]}, B_{\left[v_{L}\right]}\right)=0$. According to (3) such vectors $B_{\left[v_{L}\right]}$ have the form $\left[B v_{L}\right]$ with $B \in \mathfrak{u}(\mathcal{H})$ and

$$
0=\left\langle[A, B] v_{L} \mid v_{L}\right\rangle=-\left\langle v_{L} \mid[B, A] v_{L}\right\rangle
$$

We can choose

$$
B=i \sigma_{\alpha_{1}} \otimes \cdots \otimes \sigma_{\alpha_{L}}
$$

with $\alpha_{i} \in\{x, y, z, 0\}$ and $\sigma_{0}=1$ since such vectors span $\mathfrak{u}(\mathcal{H})$.

To prove that $T_{\left[v_{L}\right]} \mathcal{F}_{\left[v_{L}\right]}=\left(T_{\left[v_{L}\right]} \mathcal{O}_{\left[v_{L}\right]}\right)^{\perp \omega}$ we have to show that if $B_{\left[v_{L}\right]}=\left[B v_{L}\right]$ belongs to $\left(T_{\left[v_{L}\right]} \mathcal{O}_{\left[v_{L}\right]}\right)^{\perp \omega}$ then the curve $t \mapsto\left[e^{i t B} v_{L}\right]$ is contained in the fiber of the moment map, i.e.,

$$
\left\langle\mu\left(\left[v_{L}\right]\right), e^{-i t B} A e^{i t B}\right\rangle=\left\langle v_{L} \mid e^{-i t B} A e^{i t B} v_{L}\right\rangle=0,
$$

for arbitrary $A$ and $B$ of the forms, respectively, (64) and (66), fulfilling (65). To this end we employ the Hadamard lemma,

$$
e^{-i t B} A e^{i t B}=A+(-i t)[B, A]+\frac{(-i t)^{2}}{2 !}[B,[B, A]]+\frac{(-i t)^{3}}{3 !}[B,[B,[B, A]]]+\cdots .
$$

Now, using $\sigma_{\alpha_{n}}^{2}=I$ we have from (64) and (66),

$$
[B,[B, A]]=-i I \otimes \cdots \otimes\left[\sigma_{\alpha_{k}},\left[\sigma_{\alpha_{k}}, \sigma_{\beta}\right]\right] \otimes \cdots \otimes I
$$

From the commutation relations for Pauli matrices

$$
\left[\sigma_{x}, \sigma_{y}\right]=i \sigma_{z}, \quad\left[\sigma_{y}, \sigma_{z}\right]=i \sigma_{x}, \quad\left[\sigma_{z}, \sigma_{x}\right]=i \sigma_{y}
$$

we infer that the double commutator $[B,[B, A]]$ equals $A$ (possibly up to the sign) or vanishes (if $\alpha_{k}=\beta$ or $\sigma_{\alpha_{k}}=I$ ). Consequently in the expansion (68) we encounter only the terms proportional to $A$ and $[B, A]$. But $\left\langle v_{L} \mid A v_{L}\right\rangle=\frac{1}{2} \operatorname{tr} \sigma_{\beta}=0$ and $\left\langle v_{L} \mid[B, A] v_{L}\right\rangle=0$ vanishes on the assumption (65). This concludes a proof of

Fact 3 The tangent space $T_{\left[v_{L}\right]} \mathcal{F}_{\left[v_{L}\right]}$ to the fiber of the moment map over $\mu\left(\left[v_{L}\right]\right)$ is exactly equal to $\left(T_{\left[v_{L}\right]} \mathcal{O}_{\left[v_{L}\right]}\right)^{\perp \omega}$ and orbits $\mathcal{O}_{\left[v_{L}\right]}$ are isotropic. 


\section{Multiqubit systems}

In this section using geometric properties of state $\left[v_{3}\right]$ described in previous section we present easy method of checking whether two states $[u]$ and $[v]$ of three qubits are locally unitary equivalent. Notice at the beginning that in case of two qubits states the necessary and sufficient condition for this is given by equality of Schmidt decompositions. For three qubits we already know that states for which $\mu([v])=0$ are locally equivalent and lie on the orbit $\mathcal{O}_{\left[v_{3}\right]}$ which is Lagrangian. For other states the following reasoning is crucial.

Let us consider the action of $\mathcal{G}=U(\mathcal{H})$ on the complex projective space $\mathbb{P}(\mathcal{H})$. Let $x=[u]$ and $y=[v]$ be two points from $\mathbb{P}(\mathcal{H})$. Since $\mathcal{G}$ - action is transitive on $\mathbb{P}(\mathcal{H})$ there is at least one unitary matrix $U \in \mathcal{G}$ joining $x$ with $y$, i.e,

$$
[U u]=[v]
$$

Let $V \neq U$ has the property (71). Then,

$$
U x=y=V x \Rightarrow U^{-1} V \in \operatorname{Stab}(x) .
$$

Hence, there is $W \in \operatorname{Stab}(x)$ such that $V=U W$. It means that all matrices joining $x$ with $y$ are of the form $U W$ where $W \in \operatorname{Stab}(x)$. Let us consider now three vectors $v^{1}, v^{2}$ and $v^{3}$ such that

$$
\left\langle v^{i} \mid v^{i}\right\rangle=1,\left\langle v^{1} \mid v^{2}\right\rangle=0,\left\langle v^{1} \mid v^{3}\right\rangle=0
$$

i.e., all $v^{i}$ are normalized to one and $v^{2}, v^{3}$ are orthogonal to $v^{1}$. Notice that $v^{2}$ can be obtained from $v^{3}$ by action of unitary matrix $U_{1} \in \operatorname{Stab}\left(v^{1}\right)$. Hence, the general form of the unitary matrix joining $v^{2}$ with $v^{3}$ is

$$
U=U_{1} V \quad U_{1} \in \operatorname{Stab}\left(\left|v^{1}\right\rangle\right), V \in \operatorname{Stab}\left(\left|v^{2}\right\rangle\right) .
$$

In case of three qubits $\mathcal{H}=\mathbb{C}^{2} \otimes \mathbb{C}^{2} \otimes \mathbb{C}^{2}$ and the group of interest is $G=S U(3)^{\times 3}$. The direct consequence of property (74) is the following fact

Fact 4 Two locally equivalent states $[x]$ and $[y]$ are orthogonal to some state $[z]$ if and only if there exist $U \in \operatorname{Stab}([z]) \cap G$ such that $[U x]=[y]$.

Using this fact we will give a simple criterion to check the LU equivalence of two states $x=[u]$ i $y=[v]$. Let us assume at the beginning that $x$ and $y$ are already in the sorted trace form, i.e.,

$$
\mu(x)=\mu(y)=X_{1} \otimes I \otimes I+I \otimes X_{2} \otimes I+I \otimes I \otimes X_{3},
$$

where matrices $X_{i}$ are diagonal and at least one of them, e.g., $X_{1}$ has nondegenerate spectrum. Under this assumptions states $x$ and $y$ can be written in the form

$$
\begin{aligned}
& u=p_{11}|0\rangle \otimes\left|\Psi_{1}\right\rangle+p_{12}|1\rangle \otimes\left|\Psi_{2}\right\rangle, \\
& v=p_{11}|0\rangle \otimes\left|\Phi_{1}\right\rangle+p_{12}|1\rangle \otimes\left|\Phi_{2}\right\rangle,
\end{aligned}
$$


where $\left\langle\Psi_{i} \mid \Psi_{j}\right\rangle=\delta_{i j}$ and $\left\langle\Phi_{i} \mid \Phi_{j}\right\rangle=\delta_{i j}\left(\left|\Psi_{i}\right\rangle\right.$ and $\left|\Phi_{i}\right\rangle$ are two-qubit states). From (76) we see that necessary condition for $x$ and $y$ to be locally equivalent is local equivalence of pairs $\left|\Psi_{1}\right\rangle$, $\left|\Phi_{1}\right\rangle$ and $\left|\Psi_{2}\right\rangle,\left|\Phi_{2}\right\rangle$, but this can be easily checked using Schmidt decomposition as these are two-qubit states. Assume that necessary condition is fulfilled. Hence, there exists a matrix $U_{2} \otimes U_{3}$ joining state $\left|\Psi_{1}\right\rangle$ with $\left|\Phi_{1}\right\rangle$, i.e.,

$$
v^{\prime}=U_{2} \otimes U_{3} u=p_{11}|0\rangle \otimes\left|\Phi_{1}\right\rangle+p_{12}|1\rangle \otimes\left|\Psi_{2}^{\prime}\right\rangle
$$

where $\left\langle\Phi_{1} \mid \Psi_{2}^{\prime}\right\rangle=0$ and $\left|\Psi_{2}^{\prime}\right\rangle=U_{2} \otimes U_{3}\left|\Psi_{2}\right\rangle$. Notice that we can still act on $v^{\prime}$ with $\operatorname{Stab}\left(\left|\Phi_{1}\right\rangle\right) \cap K$. But from Fact 4, using assumption that $\left|\Phi_{2}\right\rangle$ is locally equivalent with $\left|\Psi_{2}^{\prime}\right\rangle$ and that both $\left|\Phi_{2}\right\rangle$ and $\left|\Psi_{2}^{\prime}\right\rangle$ are orthogonal to $\left|\Phi_{1}\right\rangle$ we obtain that $x$ is locally equivalent to $y$. Summing up, states of three qubits (76) are locally equivalent if and only if the corresponding pairs of states of two qubits $\left|\Psi_{1}\right\rangle,\left|\Phi_{1}\right\rangle$ and $\left|\Psi_{2}\right\rangle,\left|\Phi_{2}\right\rangle$ are locally equivalent. Notice that this method can be used to investigate local equivalence of states of four qubits, but only if at least one of the matrices (28) $C^{k}$ has nondegenerate spectrum. The example of the state for which all four matrices $C^{k}$ have degenerate spectrum is $\left[v_{4}\right]$. In Section $[6$ we proved that the orbit $\mathcal{O}_{\left[v_{4}\right]}$ is not lagrangian but isotropic and fiber of the moment map over $\mu\left(\left[v_{4}\right]\right)$ is not entirely contained inside the orbit $\mathcal{O}_{\left[v_{4}\right]}$. In fact the dimension of the part which is not contained in $\mathcal{O}_{\left[v_{4}\right]}$ is 12 and this makes the problem of local equivalence hard.

\section{Summary}

The presented symplectic approach to entanglement exhibited a priori unexpected geometric richness of the space of pure states for multipartite, finite dimensional quantum systems and shed some light on the important problem of the local unitary equivalence of pure states, or in physical terms, possibility of transforming one state into another by means of quantum operations restricted to single parties.

Using a fundamental concept of symplectic geometry and symplectic group action theory, viz. the moment map, the problem of the local equivalence of states is mapped from the space of states and corresponding orbits of local unitary groups onto geometry of (co)adjoint orbits in corresponding local Lie algebras and their duals. The procedure has an obvious advantage checking whether two elements of the Lie algebra or its dual space belong to the same orbit (i.e. are "locally equivalent") reduces to the comparison of spectra of (anti)symmetric matrices. On the other hand since the moment map usually is not a diffeomorphism of an orbit in the space of states onto the corresponding coadjoint orbit a detailed investigation of its fiber is needed for the ultimate check of the local equivalence of states. Such an analysis also clearly identifies situations in which a conclusive solution is hard to find.

The simplest situation occurs when an orbit of the local action in the space of states is coisotropic. In this case the whole fiber is included in the orbit and checking whether a state belongs to the orbit and hence is locally equivalent to all other states on it consists of checking if the spectra of all reduced density matrices are the same as for any other state on the orbit. However, such a situation typically occurs only in various "nondegenerate" cases. On the other hand fibers can be fully contained in the corresponding orbits also when the latter 
are not coisotropic. We have illustrated such phenomena by analyzing the bipartite case. For two particles checking of the local unitary equivalence of states can be effectively and easily done by comparison of the Schmidt spectra. This fact should be reflected in a simple geometry of local orbits. Indeed, we have shown that only when no Schmidt coefficient vanishes the orbit is cosotropic; nevertheless also non-coisotropic orbits contain the whole corresponding fibers.

In order to interpret geometrically the principal obstacles for effective checking the local unitary equivalence we analyzed the local orbits through multiqubit GHZ states. For such states all reduced density matrices are proportional to the identity (the "maximally mixed" states). The geometry of orbits through the GHZ states depends on the number of parties. For three qubits the orbit is Lagrangian, hence coisotropic. Consequently, the fiber of the moment map is contained in it which means that all states that have maximally mixed density matrices are locally unitary equivalent to the GHZ state. We also showed that if the number of qubits exceeds three the orbits through the GHZ states are isotropic rather than coisotropic, and the corresponding fibers are only partially included in them. This is the main obstacle for an easy effective checking of the local unitary equivalence.

We believe that our approach to quantum entanglement discription, although involving relatively abstract concept of symplectic geometry, has already proven to be fruitful. It not only gives an insight into geometric foundations of quantum mechanics but also contributes to the solutions of important problems of quantum information theory, hence the further continuation of this line of research seems to be very promising.

\section{Acknowledgments}

We gratefully acknowledge supports from the Polish Ministry of Science and Higher Education through the project no. N N202 090239 and the Deutsche Forschungsgemeischaft through the grant SFB-TR12.

\section{Appendix A}

We will fill some details of the calculations showing that in the two-partite case fibers of the moment map lie within the corresponding orbit. In particular we will show that the fibers are not tangent to $S$ (see (44)).

Let us define following operators

$$
X_{i j}=i\left(E_{i j}-E_{j i}\right), \quad Y_{i j}=E_{i j}+E_{j i}, \quad H_{i j}=E_{i i}-E_{j j}, \quad i<j,
$$

where $E_{i j}$ are matrices defined as

$$
\left(E_{i j}\right)_{k l}= \begin{cases}0 & \text { for } k \neq i, l \neq j \\ 1 & \text { for } k=i \mathrm{i}, l=j .\end{cases}
$$

Without losing generality we assume that $p_{1} \neq 0$ in (39). Notice that vectors from $S$ can be 
generated in the following way

$$
i p_{1}^{2} e_{k} \otimes f_{l}=\left[i Y_{1 k} \otimes Y_{1 l} v\right], \quad p_{1}^{2} e_{k} \otimes f_{l}=\left[i Y_{1 k} \otimes X_{1 l} v\right]
$$

Let us choose $A \in \mathfrak{s u}(N)$ as a diagonal traceless matrix with $A_{k k}=i$ if $p_{k} \neq 0$ and $A_{k k}=-i \frac{N-m_{0}}{m_{0}}$ if $p_{k}=0$ in (39). We have

$$
D^{1} \mu_{I \otimes A}(\ddot{x}(0))=\omega(\widehat{I \otimes A}, \ddot{x}(0))=\omega(0, \ddot{x}(0))=0 .
$$

We used $\widehat{I \otimes A}=[I \otimes A v]$ which follows from the fact that $I \otimes A v=i v$ and as such it corresponds to the zero vector in the tangent space $T_{[v]} M$. What is left to be shown is thus

$$
\dot{x}(0)^{T}\left[D^{2} \mu_{I \otimes A}\right] \dot{x}(0) \neq 0 .
$$

for any $\dot{x}(0) \in S$. Let us thus write

$$
\dot{x}(0)=\sum_{k, l}\left(a_{k l} e_{k} \otimes f_{l}+b_{k l} i e_{k} \otimes f_{l}\right)=p_{1}^{-2} \sum_{k, l}\left[i\left(a_{k l} Y_{1 k} \otimes X_{1 l}+b_{k l} Y_{1 k} \otimes Y_{1 l}\right) v\right](\text { A.6) }
$$

where the sum goes over such $k, l$ that $p_{k}=0=p_{l}$ in (39) and we used (A.3) to obtain the second equality.

To calculate explicitly the second derivative using (52) we need some commutators,

$$
\begin{aligned}
& {\left[I \otimes A, i Y_{1 k} \otimes Y_{1 l}\right]=i Y_{1 k} \otimes\left[A, Y_{1 l}\right]=i \alpha Y_{1 k} \otimes X_{1 l},} \\
& {\left[I \otimes A, i Y_{1 k} \otimes X_{1 l}\right]=i Y_{1 k} \otimes\left[A, X_{1 l}\right]=-i \alpha Y_{1 k} \otimes Y_{1 l},}
\end{aligned}
$$

where $\alpha=\frac{N}{m_{0}}$. Hence,

$$
\left[I \otimes A, \sum_{k, l} i\left(a_{k l} Y_{1 k} \otimes X_{1 l}+b_{k l} Y_{1 k} \otimes Y_{1 l}\right)\right]=i \alpha \sum_{k, l} b_{k l} Y_{1 k} \otimes X_{1 l}-a_{k l} Y_{1 k} \otimes Y_{1 l}
$$

And, finally,

$\dot{x}(0)\left[D^{2} \mu_{I \otimes A}\right] \dot{x}(0)=$

$=-i\left\langle\left[\left[I \otimes A, p_{1}^{-2} \sum_{k, l} i\left(a_{k l} Y_{1 k} \otimes X_{1 l}+b_{k l} Y_{1 k} \otimes Y_{1 l}\right)\right], p_{1}^{-2} \sum_{k, l} i\left(a_{k l} Y_{1 k} \otimes X_{1 l}+b_{k l} Y_{1 k} \otimes Y_{1 l}\right)\right] v \mid v\right\rangle=$

$=-i\left\langle\left[i \alpha p_{1}^{-2} \sum_{k, l}\left(b_{k l} Y_{1 k} \otimes X_{1 l}-a_{k l} Y_{1 k} \otimes Y_{1 l}\right), i p_{1}^{-2} \sum_{k, l}\left(a_{k l} Y_{1 k} \otimes X_{1 l}+b_{k l} Y_{1 k} \otimes Y_{1 l}\right)\right] v \mid v\right\rangle=$

$=-i \alpha \cdot \omega\left(\sum_{k l} b_{k l} e_{k} \otimes f_{l}-a_{k l} i e_{k} \otimes f_{l}, \sum_{k l} a_{k l} e_{k} \otimes f_{l}+b_{k l} i e_{k} \otimes f_{l}\right)=-2 i \alpha \sum_{k l}\left(a_{k l}^{2}+b_{k l}^{2}\right)$.

This clearly means that $\dot{x}(0)\left[D^{2} \mu_{I \otimes A}\right] \dot{x}(0) \neq 0$ for any $\dot{x}(0) \in S$ and proves that in the bipartite case fibers of the moment map are fully contained in the corresponding orbits.

\section{Appendix B. Two Qutrits}

In case of two qutrits $(N=3, L=2)$ the Hilbert space is $\mathcal{H}=\mathbb{C}^{3} \otimes \mathbb{C}^{3}$ and $\operatorname{dim}(\mathcal{H})=18$, so $\operatorname{dim}(\mathbb{P}(\mathcal{H}))=16$. The Lie algebra $\mathfrak{g}=\mathfrak{s u}(3) \oplus \mathfrak{s u}(3)$ of $G=S U(3) \times S U(3)$ is spanned by $\left\{A_{k} \otimes I, I \otimes A_{k}\right\}$, where $\left\{A_{k}, k=1, \ldots, 8\right\}$ is a basis in $\mathfrak{s u}(3)$ hence $\operatorname{dim}(\mathfrak{g})=16$. The fibers 
of moment map through $v$ are given as common level set of sixteen functions $\mu_{A_{k} \otimes I}, \mu_{I \otimes A_{k}}$. Without loosing generality we assume that the bases $\left\{e_{k}\right\}$ and $\left\{f_{k}\right\}$ in both Hilbert spaces are equal. As previously we switch to the customary notation $e_{1}=|0\rangle=f_{1}, e_{2}=|1\rangle=f_{2}$, $e_{3}=|2\rangle=f_{3}$, together with $|k l\rangle=|k\rangle \otimes|l\rangle$.

The general form of a Schmidt-decomposed two-qutrit state is given by

$$
v=p_{1}|00\rangle+p_{2}|11\rangle+p_{3}|22\rangle,
$$

where $p_{1}^{2}+p_{2}^{2}+p_{3}^{2}=1$. There are six cases to consider.

\section{1. $p_{1}=1, p_{2}=p_{3}=0$ (a separable state)}

In this case $v=|00\rangle$. The orbit $\mathcal{O}_{[v]}$ through $[v]$ is symplectic ([1]) hence the part of the fiber which is contained in $\mathcal{O}_{[v]}$ is zero dimensional. Orthogonal complement $\left(T_{[v]} \mathcal{O}_{[v]}\right)^{\perp \omega}$ is spanned by

$$
\{|22\rangle, i|22\rangle,|11\rangle, i|11\rangle,|12\rangle, i|12\rangle,|21\rangle, i|21\rangle\}
$$

and is a symplectic vector space $S$. The matrix $A \in \mathfrak{s u}(3)$ used in the proof in Appendix A has the form

$$
A=\left(\begin{array}{ccc}
i & 0 & 0 \\
0 & -\frac{i}{2} & 0 \\
0 & 0 & -\frac{i}{2}
\end{array}\right) .
$$

There is no fiber and the orbit is not coisotropic.

\section{2. $p_{1}=p_{2}=p_{3}=\frac{1}{\sqrt{3}}$ (the maximally entangled state)}

In this case the orbit $\mathcal{O}_{[v]}$ through $v=\frac{1}{\sqrt{3}}(|00\rangle+|11\rangle+|22\rangle)$ is coisotropic since all $p_{k} \neq 0$. In fact $\mathcal{O}_{[v]}$ is minimally coisotropic hence Lagrangian, i.e.,

$$
\begin{aligned}
& \left(T_{[v]} \mathcal{O}_{[v]}\right)^{\perp \omega}=T_{[v]} \mathcal{O}_{[v]}, \\
& \operatorname{dim}\left(T_{[v]} \mathcal{O}_{[v]}\right)=\frac{1}{2} \operatorname{dim} \mathbb{P}(\mathcal{H}) .
\end{aligned}
$$

Using formula (43) it is easy to prove that in case of two qunits it is always true that orbit through

$$
v=\sum_{k=1}^{N} \frac{1}{\sqrt{N}}|k k\rangle,
$$

is Lagrangian. Namely for (A.15) we have

$$
\left(T_{[v]} \mathcal{O}_{[v]}\right)^{\perp \omega}=D([v])=N^{2}-1=\frac{1}{2} \operatorname{dim} \mathbb{P}(\mathcal{H}),
$$

hence $\mathcal{O}_{[v]}$ is Lagrangian [8]. 
3. $p_{1} \neq p_{2} \neq p_{3} \neq 0$ (a generic state)

The orbit $\mathcal{O}_{[v]}$ through $v=p_{1}|00\rangle+p_{2}|11\rangle+p_{3}|22\rangle$ is coisotropic since all $p_{k} \neq 0$. Formulas (40) and (41) give

$$
\operatorname{dim}\left(\mathcal{O}_{[v]}\right)=14, \operatorname{dim}\left(\mu\left(\mathcal{O}_{[v]}\right)\right)=12 .
$$

The whole is fiber is contained in $\mathcal{O}_{[v]}$ and is two-dimensional.

4. $p_{1}=p_{2} \neq 0, \quad p_{3} \neq 0$

The orbit $\mathcal{O}_{[v]}$ through $v=p_{1}(|00\rangle+|11\rangle)+p_{3}|22\rangle$ is coisotropic since all $p_{k} \neq 0$. Formulas (40) and (41) give

$$
\operatorname{dim}\left(\mathcal{O}_{[v]}\right)=12, \operatorname{dim}\left(\mu\left(\mathcal{O}_{[v]}\right)\right)=8
$$

The whole fiber is contained in $\mathcal{O}_{[v]}$ and is four-dimensional.

5. $p_{1}=p_{2}=\frac{1}{\sqrt{2}}, \quad p_{3}=0$

The orbit $\mathcal{O}_{[v]}$ through $v=\frac{1}{\sqrt{2}}(|00\rangle+|11\rangle)$ is not coisotropic since $p_{3}=0$. Formulas (40) and (41) give

$$
\operatorname{dim}\left(\mathcal{O}_{[v]}\right)=11, \operatorname{dim}\left(\mu\left(\mathcal{O}_{[v]}\right)\right)=8
$$

Hence the part of the fiber contained in $\mathcal{O}_{[v]}$ is three-dimensional. The orthogonal complement $\left(T_{[v]} \mathcal{O}_{[v]}\right)^{\perp \omega}$ is five-dimensional and is spanned by three vectors contained in $T_{[v]} \mathcal{O}_{[v]}$ and two other $\left\{v_{1}=|22\rangle, v_{2}=i|22\rangle\right\}$. The matrix $A \in \mathfrak{s u}(3)$ used in the proof in Appendix A has the form

$$
A=\left(\begin{array}{ccc}
i & 0 & 0 \\
0 & i & 0 \\
0 & 0 & -2 i
\end{array}\right)
$$

The whole fiber is contained inside the orbit although the orbit is not coisotropic.

6. $p_{1} \neq p_{2} \neq 0, \quad p_{3}=0$

The orbit $\mathcal{O}_{[v]}$ through $v=p_{1}|00\rangle+p_{2}|11\rangle$ is not coisotropic since $p_{3}=0$. Formulas (40) and (41) give

$$
\operatorname{dim}\left(\mathcal{O}_{[v]}\right)=13, \operatorname{dim}\left(\mu\left(\mathcal{O}_{[v]}\right)\right)=12
$$

Hence the part of the fiber contained in $\mathcal{O}_{[v]}$ is one-dimensional. The orthogonal complement $\left(T_{[v]} \mathcal{O}_{[v]}\right)^{\perp \omega}$ is three-dimensional and is spanned by one vector contained in $T_{[v]} \mathcal{O}_{[v]}$ and two 
other $\left\{v_{1}=|22\rangle, v_{2}=i|22\rangle\right\}$. The matrix $A \in \mathfrak{s u}(3)$ used in the proof in Appendix A has the form

$$
A=\left(\begin{array}{ccc}
i & 0 & 0 \\
0 & i & 0 \\
0 & 0 & -2 i
\end{array}\right)
$$

Again the whole fiber is contained inside the orbit although the orbit is not coisotropic.

\section{References}

[1] Sawicki A, Huckleberry A and Kuś M 2011 Symplectic geometry of entanglement. Commun. Math. Phys., 305(2):441-468

[2] Kraus B 2010 Local unitary equivalence of multipartite pure states. Phys. Rev. Lett., 104(2):020504

[3] Kraus B 2010 Local unitary equivalence and entanglement of multipartite pure states. arXiv 1005.5295

[4] Guillemin V and Sternberg S 1984 Symplectic techniques in physics. Cambridge University Press

[5] Kirillov A A 2004 Lectures on the orbit method, volume 64 of Graduate Studies in Mathematics. American Mathematical Society

[6] Kostant B and Sternberg S 1982 Symplectic projective orbits. In P. J. Hilton and G. S. Young, editors, New directions in applied mathematics: papers presented April 25/26, 1980, on the occasion of the Case Centennial Celebration, New York, Springer-Verlag

[7] Sinołęcka M M, Zyczkowski K and Kuś M 2002 Manifolds of equal entanglement for composite quantum system. Acta Phys. Pol., 33:2081

[8] Bengtsson I 2007 A curious geometrical fact about entanglement. arXiv 0707.3512 